\title{
Unconventional Microwave Source
}

\author{
Bucur M. Novac, Senior member, IEEE, Peter Senior, Member, IEEE, Michael Farage, Ivor R. Smith, \\ Senior Member, IEEE and Renzhen Xiao
}

\begin{abstract}
A number of published papers are reporting that electromagnetic radiation is being generated by unexpected sources such as explosives, metal fracturing or peeling common adhesive tape under vacuum. Here we report on an unconventional microwave source (UMS) that produces, with a high degree of reproducibility, electromagnetic radiation covering an unusually wide frequency spectrum ranging from hundreds of $\mathrm{kHz}$ to many tens of GHz. The UMS is based on picosecond electric breakdown of a polymer and does not require vacuum technology.
\end{abstract}

Index Terms-Antenna, microwave generators

\section{INTRODUCTION}

A NUMBER of papers published previously are dedicated to the electromagnetic radiation generated by unexpected sources, such as explosives [1], metal fracturing [2] or peeling common adhesive tape under vacuum [3]. Here we report on an unconventional microwave source (UMS). The UMS is based simply on the electrical breakdown of a polymer in ambient air using a pin-plane metallic electrode configuration, with a dc source applying high-voltage to the pin and the plane electrode earthed, as presented schematically in Fig. 1.

However, for the UMS phenomenon to be generated, it is essential for the charged pin electrode to be mechanicallydriven into the polymer until electrical breakdown occurs when the pin is almost through.

Sources that operate on the principle of exciting 'electrically short' dipoles are described in open literature [4], [5], but the results show a limited bandwidth that it is not covering the multi-GHz region. There is however a range of pulsed $\mathrm{THz}$ sources that produce radiation over hundreds of $\mathrm{GHz}$ bandwidth using femtosecond laser excitation of photoconductors [6], [7].

Referring to Fig. 1, the UMS used in this work has a HV pin copper electrode of diameter $\mathrm{D}=10 \mathrm{~mm}$, length $\mathrm{L}=70$ $\mathrm{mm}$, a cone angle $\alpha=90$ degrees and with a tip radius of about $50 \mu \mathrm{m}$. The $\mathrm{HV}$ electrode is charged to $5 \mathrm{kV}$ and separated from an earthed copper sheet electrode by a $1.4 \mathrm{~mm}$ thick HDPE polyethylene sheet. According to the manufacturer, the material has a mass density of $0.95 \mathrm{~g} / \mathrm{cm}^{3}$,

Manuscript submitted for review on October 15, 2017.

Bucur M. Novac, Peter Senior, Michael Farage and Ivor R. Smith are with Loughborough University, Loughborough, Leicestershire LE11 3TU, UK. (correspondence e-mail: b.m.novac@lboro.ac.uk).

Renzhen Xiao is with the Northwest Institute of Nuclear Technology, Xi'an 710024, P.R. China (e-mail: xiaorenzhen@tsinghua.org.cn). a relative permittivity of 2.35 and a dielectric strength of $22 \mathrm{kV} / \mathrm{mm}$. The microwave detector, either a horn antenna or a D-dot probe, is positioned at a distance $\mathrm{S}$ from the $\mathrm{HV}$ electrode axis larger than $2 \mathrm{~L}^{2} f / c$, where $c$ is the speed of light in vacuum, such that the region where the detection takes place can be considered as being 'far field' at the maximum frequency $f$ of interest for that particular arrangement. To generate microwaves the HV electrode is firstly dc charged, with a mechanical force $\mathbf{F}$ subsequently applied to drive the electrode through the polymer until electrical breakdown is produced between the HV electrode and the earthed metallic plate. Full details on the experimental and diagnostic techniques used throughout this work are provided in the next section.

\section{DETAILS OF TECHNIQUES USED DURING THE RESEARCH}

A. Techniques used to mechanically-drive the pin charged electrode into the polymer.

The following methods have been all successfully used:

- Using an energized electromagnet, a metallic cylindrical 'anvil' is suspended at the upper part of a long dielectric tube. By interrupting the current flowing through the electromagnet, the anvil drops and hits the HV pin electrode. All the results presented in this paper were obtained using this technique. The metallic anvil must however be insulated from the pin electrode using a layer of plastic, otherwise, due to friction with the dielectric tube, the charge accumulated on the anvil may interfere with the experiment. Because of this perturbing phenomenon, during the early days of the investigation radiation was observed even without externally charging the pin, with the necessary initial electrostatic energy obtained from the charge transferred to the pin electrode from the electrified anvil.

- Systems using either a high-pressure gas bottle or the explosive charge of a detonator can both be used to accelerate the $\mathrm{HV}$ pin electrode

- Finally, the HV pin electrode can be moved simply by manually-operated mechanics.

B. Diagnostic techniques used for measuring and analyzing the electromagnetic radiation

Three types of electromagnetic sensors were used: a D-dot probe and two antennas. 


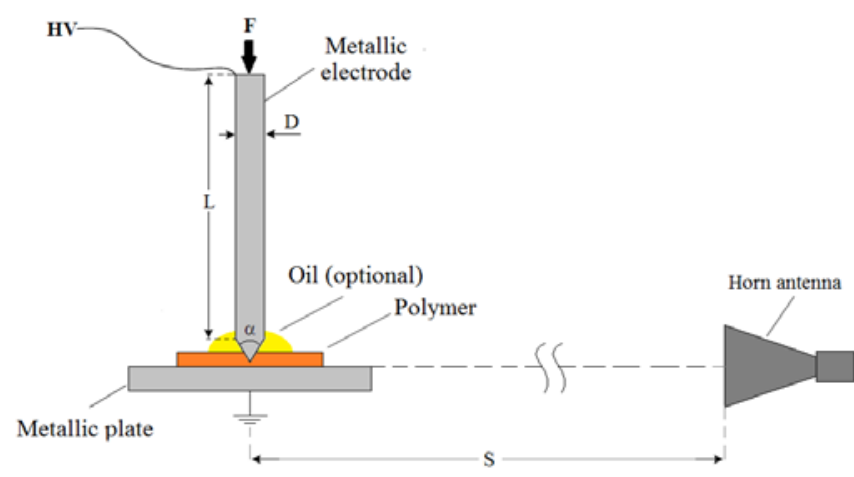

Fig. 1. Schematic of UMS, with microwave emission detected by a horn antenna (see text).

- The D-dot probe type AD-70 was used coupled to a balun to match its impedance to the $50 \Omega$ impedance of the output coaxial cable, both items manufactured by Prodyn Technologies. The D-dot generates a voltage signal $V_{D d o t}$ that is proportional to the time rate-of-change of the applied electric field $\mathrm{E}$ :

$V_{\text {Ddot }}=Z_{\text {Ddot }} A_{\text {eff }} \frac{d D}{d t} \cos \theta$

where $Z_{D d o t}=100 \Omega$ is the probe impedance, $A_{e f f}=10^{-3} \mathrm{~m}^{2}$ is its effective area, $D=\varepsilon_{0} E$ is the displacement field in air and $\theta$ is the angle between the electric field vector and the normal to the probe ground plane. In all experiments, knowledge of the direction of the vector $E$ allowed positioning the probe such that $\cos \theta=1$. The signal from the probe is attenuated by $8 \mathrm{~dB}$ in the balun and, as with the antennas used in this work, use of supplementary attenuators allows the output signal to be recorded by a battery-powered oscilloscope housed in a Faraday cage. Integration of the oscilloscope signal provides the time history of the incident electric field and its FFT provides the spectral density.

- Both dual polarized horn antennas used in this work, DP240 and DP241, are manufactured by Flann Microwaves. The frequency analysis of their output voltage signals is performed in two steps: firstly, the FFT of the antenna signal is calculated and secondly the electric field antenna factor (EFAF) is used to obtain the incident electric field spectrum. The frequency dependent $\operatorname{EFAF}(f)$ for the two antennas presented in Fig. 2 is obtained from:

$\operatorname{EFAF}(f)=2 f \sqrt{\frac{\pi \mu_{0}}{c Z_{\text {load }} G(f)}}$

where $Z_{\text {load }}=50 \Omega$ is the input impedance of the oscilloscope and $G(f)$ is the frequency dependent antenna gain provided by the manufacturer.

\section{MAJOR FINDINGS}

The following major characteristics of the UMS have been demonstrated experimentally:

- The initial energy of the UMS is stored in the interelectrode capacitance during dc charging. Due to the extremely short time scale during which radiation is emitted,

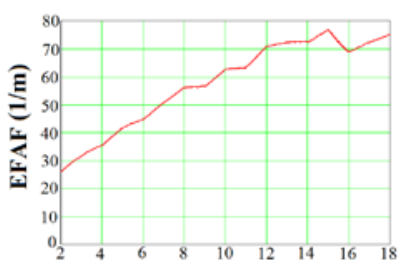

frequency $(\mathrm{GHz})$

a)

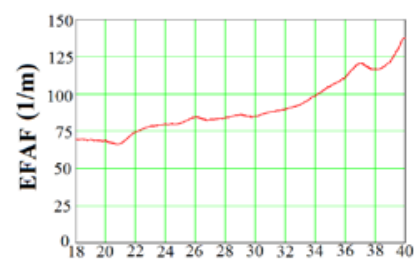

frequency $(\mathrm{GHz})$

b)
Fig. 2. Electric field antenna factor for a) DP240 and b) DP241.

the external charging circuitry with its relatively large selfinductance and capacitance plays no role, because of its much longer characteristic time. As the initial energy at a charge voltage of $5 \mathrm{kV}$ is extremely small i.e., of the order of a few tens of micro Joules, the inter-electrode capacitance can also be charged by unexpected and uncontrolled electrostatic processes, such as electrostatic charging by friction of parts of the mechanism used to mechanically drive the high-voltage pin electrode. When the electromagnetic radiation emitted from a dielectric closing switch of a capacitor bank was studied [8], the electrostatic charging complicated the matters (details were provided above).

- Figs. 3, 4 and 5 show the time domain and the FFT characteristics of the electromagnetic radiation emitted by the UMS when charged at $5 \mathrm{kV}$, having an exceptionally large bandwidth ranging from $200 \mathrm{kHz}$ to $40 \mathrm{GHz}$. To obtain these results the measurements used the three sensors presented above, coupled to various oscilloscopes, as indicated in the corresponding figures captions. The electromagnetic radiation emitted by UMS appears to be generated by two different processes: a first and extremely fast process termed 'Spike', followed by lower frequency damped oscillations similar to those generated by a RLC discharge (Figs. 4 (b) and 5 (b)).

- The electromagnetic wave is polarized, with the vertically-polarized component parallel to the HV electrode axis more than four times more intense than the horizontallypolarized component. An example is provided in Fig. 6.

- Altering the inter-electrode capacitance by various techniques, for example surrounding the HV pin electrode by a drop of oil as suggested in Fig. 1, changes the frequency and amplitude of the damped oscillations (as expected) but leaves the Spike process unaffected. An example is provided in Fig 7.

- The UMS phenomenon takes place irrespective of the polarity of the applied dc voltage.

- For charging voltages between $\pm 5 \mathrm{kV}$ and $\pm 30 \mathrm{kV}$ the radiation intensity of UMS scales almost linearly with the voltage applied i.e., with the peak electrostatic field generated in the dielectric material. Results for measurements performed with a $3.5 \mathrm{GHz}$ D-dot sensor positioned at $1.2 \mathrm{~m}$ distance (an example is provided in Fig. 3 at $5 \mathrm{kV}$ charging voltage), indicated that the transient peak electric field can be conveniently expressed as: 


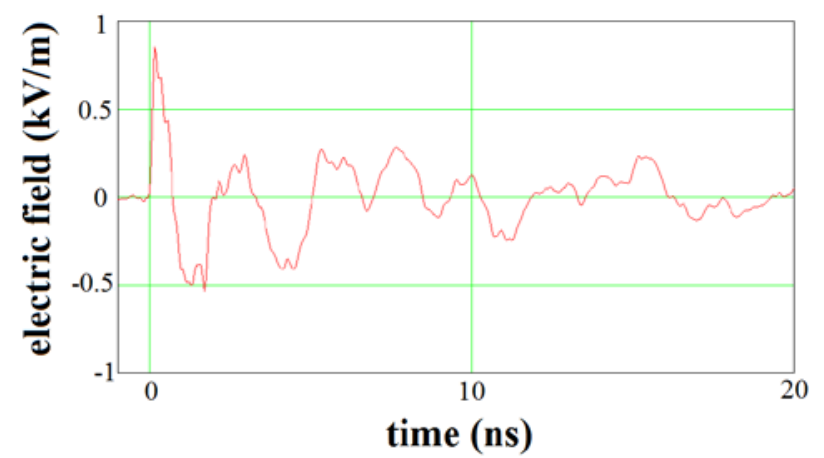

a)

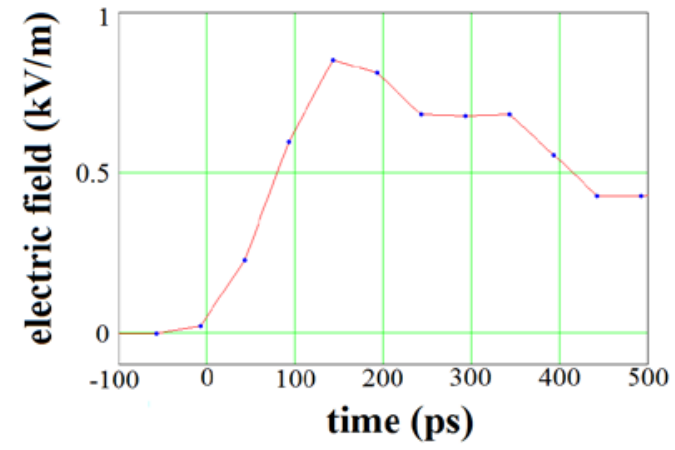

b)

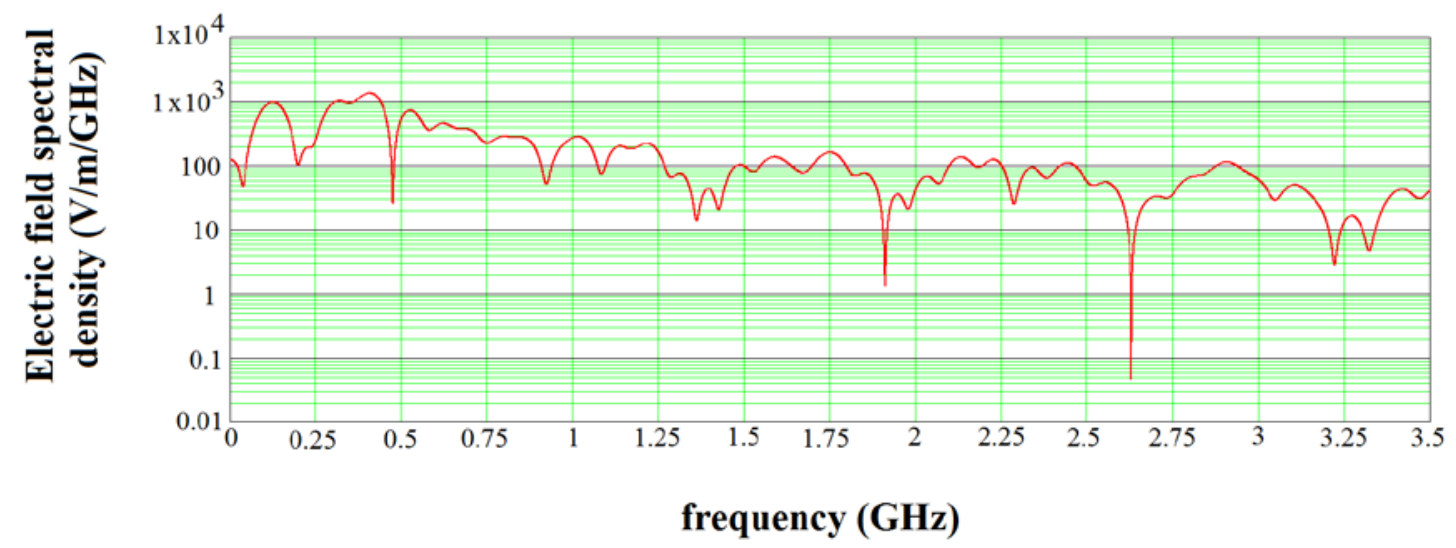

c)

Fig. 3. Microwave radiation emitted by UMS and detected with a $3.5 \mathrm{GHz}$ D-dot probe. The D-dot probe (Prodyn AD-70) was positioned $1.2 \mathrm{~m}$ from the $\mathrm{HV}$ electrode and, when used with its dedicated balun, has a bandwidth ranging from $200 \mathrm{kHz}$ to $3.5 \mathrm{GHz}$. The real time digital oscilloscope used to record the signal has a $6 \mathrm{GHz}$ analogue bandwidth with $20 \mathrm{GS} / \mathrm{s}$. a) Typical vertical polarization electric field signal (obtained by numerical integrating the differential recorded signal). b) the first $500 \mathrm{ps}$, with dots showing the real digital signal and a time interval of 50 ps between consecutive dots. c) Spectral density of the incident electric field corresponding to a).

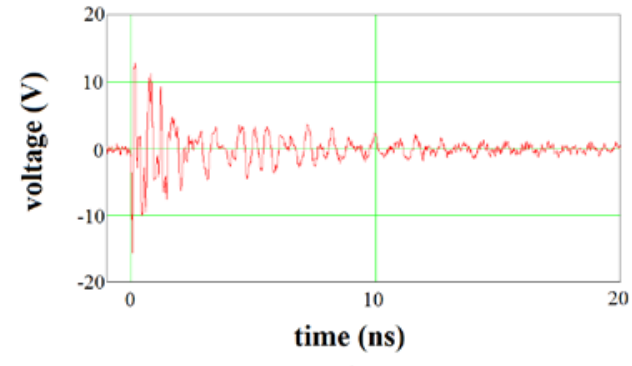

a)

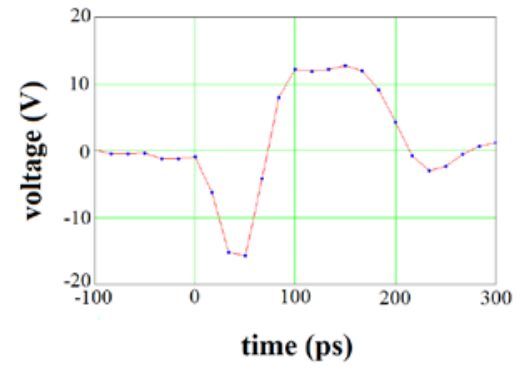

b)

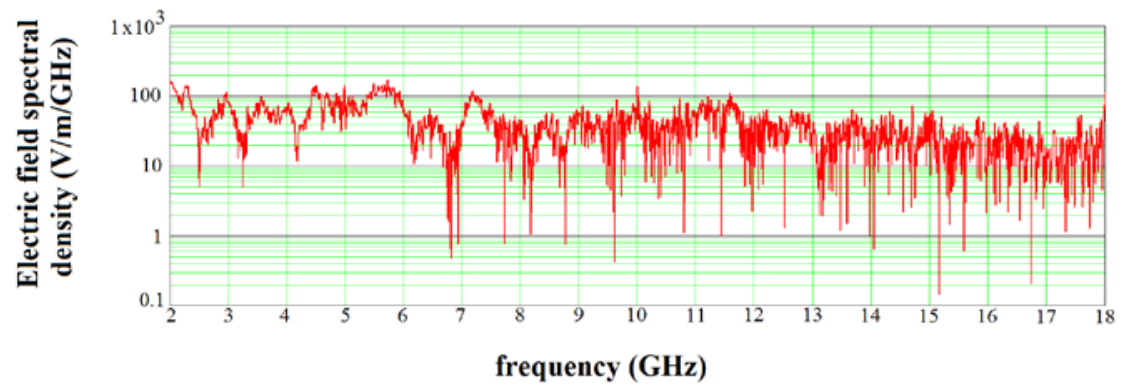

c)

Fig. 4. Microwave radiation emitted by UMS and detected with a $18 \mathrm{GHz}$ horn antenna. The dual polarized antenna (Flann DP240) was positioned $1.7 \mathrm{~m}$ from the $\mathrm{HV}$ electrode and has a bandwidth ranging from $2 \mathrm{GHz}$ to $18 \mathrm{GHz}$. The real time digital oscilloscope used to record the signal has an analogue bandwidth of $18 \mathrm{GHz}$ with $60 \mathrm{GS} / \mathrm{s}$.

a) Typical vertical polarization voltage signal from antenna. b) the first $300 \mathrm{ps}$, with dots showing the real digital signal with a time interval of 16.33 ps between consecutive dots. c) Spectral density of the incident electric field corresponding to a). 


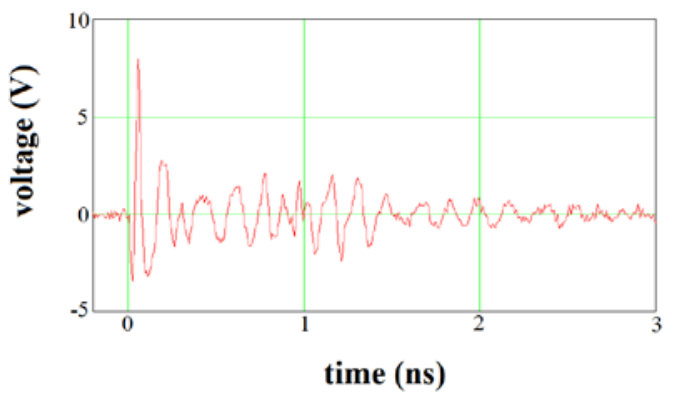

a)

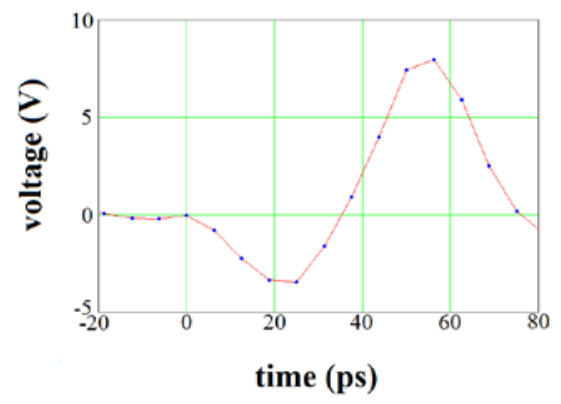

b)
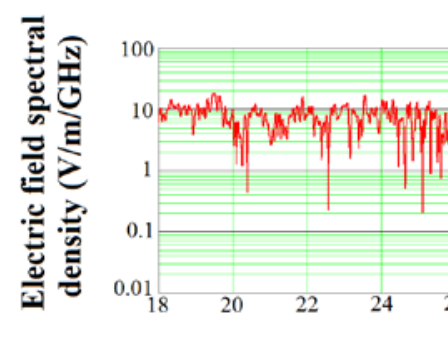

\section{frequency $(\mathrm{GHz})$}

c)

Fig. 5. Microwave radiation emitted by UMS and detected with a $40 \mathrm{GHz}$ horn antenna. The dual polarized antenna (Flann DP241) was positioned $1.4 \mathrm{~m}$ from the HV electrode and has a bandwidth ranging from $18 \mathrm{GHz}$ to $40 \mathrm{GHz}$. The real time digital oscilloscope used to record the signal has an analogue bandwidth of $65 \mathrm{GHz}$ with $160 \mathrm{GS} / \mathrm{s}$. a) Typical vertical polarization voltage signal. b) the first $80 \mathrm{ps,} \mathrm{with} \mathrm{dots} \mathrm{showing} \mathrm{the} \mathrm{real} \mathrm{digital}$ signal with a time interval of 6.25 ps between consecutive dots. c) Spectral density of the incident electric field corresponding to a).

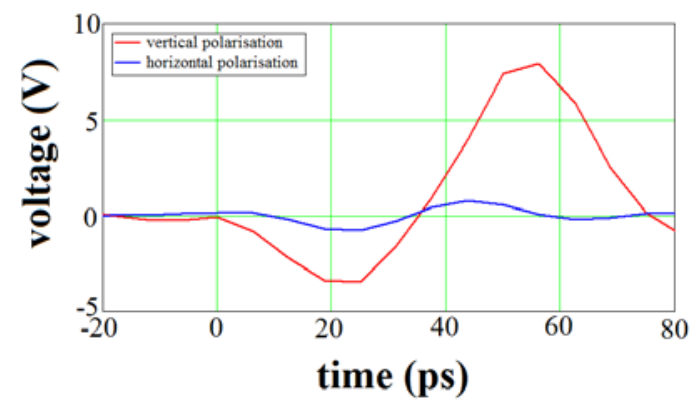

Fig. 6. Polarization of the microwave radiation. The data for the vertical polarization (along the pin electrode) is the same as in Fig. 5.b. For comparison, the signal corresponding to horizontal polarization is also presented.

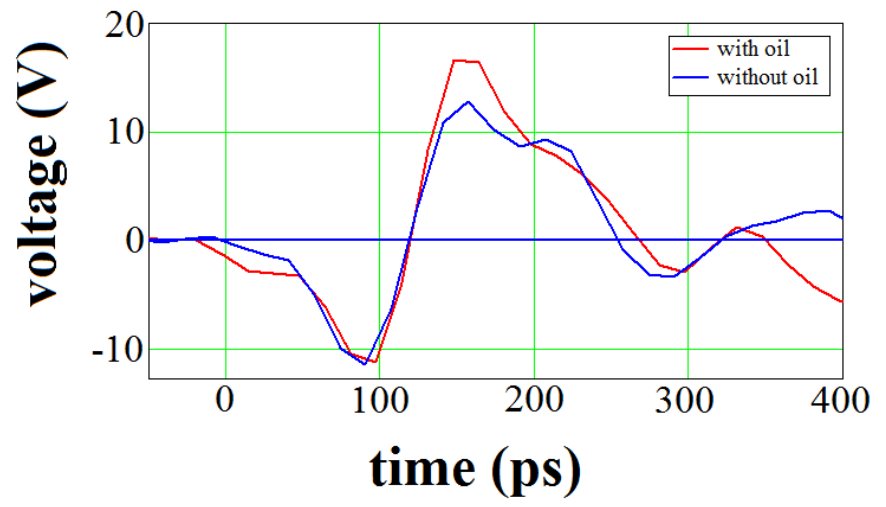

Fig. 7. Microwave radiation emitted is enhanced by a drop of oil (see Fig. 1). In both cases, with or without oil, the fast negative 'Spike' signal emitted is the same. However, the first positive peak of the damped oscillation is clearly enhanced by the presence of oil. The antenna (Flann DP240) is positioned $1.7 \mathrm{~m}$ from the HV electrode. The real time digital oscilloscope used to record the signal has an analogue bandwidth of $18 \mathrm{GHz}$ with $60 \mathrm{GS} / \mathrm{s}$. 


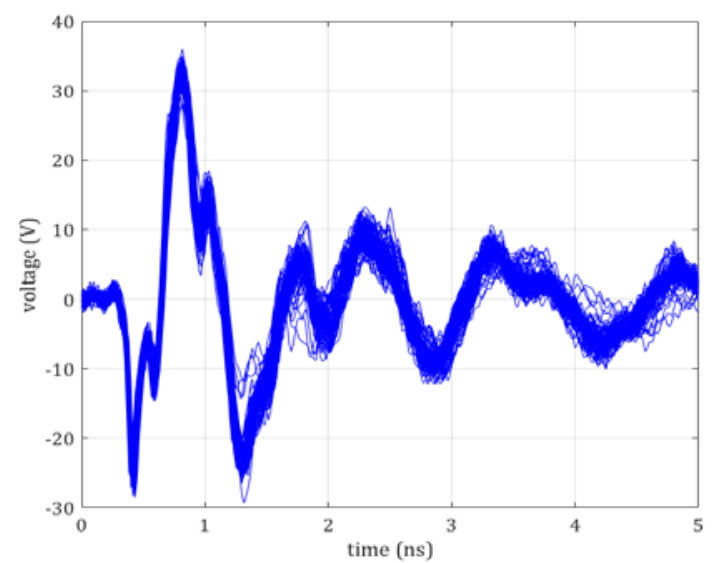

Fig. 8. UMS reproducibility. The data shown is from 100 'identical' shots as recorded i.e., without any time adjustment. The antenna (Flann DP240) is positioned $1.4 \mathrm{~m}$ from the HV electrode. The real time digital oscilloscope used to record the signal has an analogue bandwidth of $18 \mathrm{GHz}$ with $60 \mathrm{GS} / \mathrm{s}$.

$$
\mathrm{E}_{\text {peak }}(\mathrm{U})=0.18 \cdot|\mathrm{U}|+2 \cdot 10^{-4} \cdot \mathrm{U}^{2}
$$

where $\mathrm{E}_{\text {peak }}$ is in $\mathrm{kV} / \mathrm{m}$ and the charging voltage $\mathrm{U}$ is in $\mathrm{kV}$.

- The UMS phenomenon has extremely good reproducibility, with results from 100 'identical' shots presented in Fig. 8 being practically indistinguishable.

- The UMS phenomenon can be generated using a large number of different solid dielectric materials including Mylar, Kapton, Teflon and polyethylene, with the best results so far having been obtained when using polyethylene. None of the samples used had any adhesive film applied nor have been annealed or altered in any way. Polyethylene samples obtained from various companies provided the same results i.e, the precise composition seems to play no role.

- Liquid insulators such as oil, glycerin or propylene carbonate, do not generate the phenomenon

- Samples of thin ceramics were also investigated, but no UMS radiation was ever detected.

- The thickness of the polymer plays no role, suggesting that breakdown takes place only when the pin-plate interelectrode distance is very small, perhaps of the order of a few tens of microns. As a consequence, the geometrical enhancement feature of the electric fields can give rise to intensities of many $\mathrm{MV} / \mathrm{cm}$. This probably triggers what is termed an 'intrinsic' breakdown [9] and may provide an explanation for the remarkable reproducibility of the UMS radiation pattern.

- Experiments have been performed with the HDPE polyethylene sample maintained at various temperatures. Although the manufacturer catalogue indicates $120^{\circ} \mathrm{C}$ as the maximum 'working temperature', tests have been performed at even higher temperatures, with the melting point of the material being close to $160{ }^{\circ} \mathrm{C}$. It was found that the temperature of the dielectric material, tested in the range from $-5^{\circ} \mathrm{C}$ to $150^{\circ} \mathrm{C}$, does not have any influence on the UMS.

- The magnitude of the force ( $\mathbf{F}$ in Fig. 1 ) acting on the dynamic HV pin electrode appears not to influence the results, providing that it is sufficient to advance the pin through the dielectric until electric breakdown is produced.

- The influence of the metallic pin electrode diameter D (see Fig. 1) was also investigated. It was found that by increasing the diameter two times, the intensity of radiation detected by a D-dot probe is enhanced by $50 \%$. That can easily be explained by an increase in the inter-electrode capacitance due to a larger electrode conical tip.

\section{NUMERICAL MODELING}

In the models described below, the electrode will be considered as a dipole antenna excited with a fast voltage step impulse. The initial energy is stored as electrostatic energy in the electrode-ground plate capacitor. As an electric breakdown in a solid dielectric usually occurs very fast (at ps time scale), the discharge of the antenna-capacitor configuration will generate a step-like voltage impulse input. Since an antenna can only radiate ac signals, the antenna will act like a differentiating element to the voltage step input. This may explain the 'Spike' effect.

\section{A. PSpice modeling}

The energy transfer mechanism in the system can be studied with the aid of the simplest and low-frequency model, with the equivalent electric circuit of the system shown in Fig. 9 and a corresponding PSpice model (not shown). The metallic electrode (antenna) is modelled as a multi-stage pulse forming network. The self-inductance value $\mathrm{L}_{0}=1.5 \mathrm{nH}$ has been estimated from a simple formula, while the capacitance values $\mathrm{C}_{0}=0.6 \mathrm{pF}$ for the network and $\mathrm{C}_{\text {gap }}=1.68 \mathrm{pF}$ for the spark gap were both obtained from modeling using an electrostatic solver (Ansys Maxwell $\mathrm{SV}$ ). The term $\mathrm{L}_{\mathrm{g}}=18 \mathrm{nH}$ includes both the self-inductance of the breakdown arc channel and that corresponding to the current path along the metallic plate towards the remote ground. The energy lost by radiation is taken into account by the antenna impedance $\mathrm{R}_{\mathrm{ant}}=30 \Omega$. Both $\mathrm{L}_{\mathrm{g}}$ and $\mathrm{R}_{\mathrm{ant}}$ have their values adjusted for matching the calculated form of the voltage across the antenna with the experimentally detected electric signal. The model considers the initial 


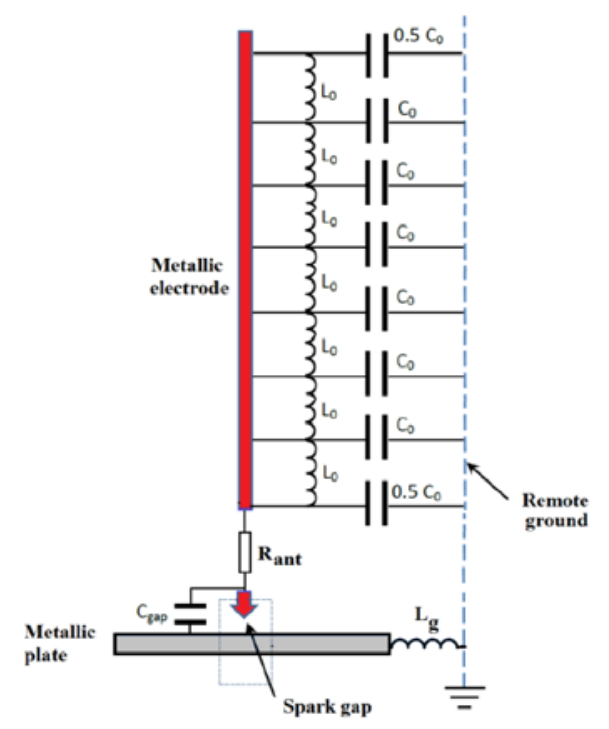

Fig. 9. Low-frequency model of the UMS: equivalent electric circuit of the system (see text).

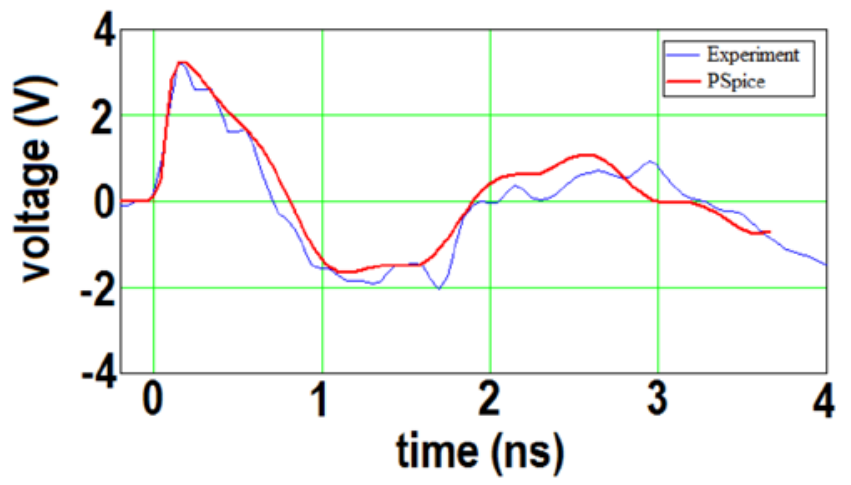

Fig. 10. Qualitative comparison between the antenna voltage predicted by the low-frequency model and the time variation of the electric field measured using a D-dot probe. For clarity, the voltage waveform has been scaled to match the electric field.

electrostatic energy stored in both $\mathrm{C}_{\text {gap }}$ and in the stray capacitances $\mathrm{C}_{0}$, all initially charged to $5 \mathrm{kV}$. The switch resistance was chosen to drop linearly from $1 \mathrm{M} \Omega$ to $50 \Omega$ in $600 \mathrm{ps}$ and from $50 \Omega$ to $10 \mathrm{~m} \Omega$ in $3 \mathrm{ps}$, the latter representing the estimated switching time. Fig. 10 shows the model allows a very close qualitative matching between the calculated antenna voltage and the measured electric field. The sinusoidal-like current flowing through the antenna (not shown) gives an indication for the input data required for the more complex modelling using CST presented below.

\section{CST modeling}

The CST Microwave Studio model of the UMS system can be seen in Fig. 11. In the real experiment the ground plate potential is not floating. As CST does not allow this, a method to fix the potential of the ground plate to $0 \mathrm{~V}$ is to consider a mirror $\mathrm{HV}$ electrode charged to an opposite polarity voltage as in Figs 11(a) and 11(b), with the XZ plane of symmetry having the tangential component of the electric field $\mathrm{E}_{\mathrm{t}}=0$ (Fig. 11(c)). For maintaining the calculation time at a practical level (a complete simulation takes about 10 hours on a $2.6 \mathrm{GHz}, 256 \mathrm{~Gb}$ RAM work station), a finite element mesh grid of $200 \mu \mathrm{m}$ was considered inside the polyethylene volume between the two metallic elements separated by $200 \mu \mathrm{m}$ (see Fig. 10(c)). The tip radius of the metallic electrodes is $50 \mu \mathrm{m}$ (same as in real experiments), with the calculation performed with a time step of about $1 \mathrm{ps}$. As there is no closing switch available in the CST software, the action of the spark gap is simulated by setting up an excitation signal of current raising in 1 ps to a peak of about $31 \mathrm{~A}$ across an equivalent resistance connected to the two tips of about $320 \Omega$. Changing the current rise time from 1 ps up to $10 \mathrm{ps}$ provides similar results, but those in the lower figure are preferred as generating data closer to experiment. The CST prediction of the spectral density in Fig. 12 shows a good correlation with the experimental data up to about $30 \mathrm{GHz}$. The frequency corresponding to the dip in the CST spectral density at around $30 \mathrm{GHz}$ decreases with the length of the electrode and increases with distance to the UMS source at which the calculations are made.

\section{B. Efficiency of generating microwave radiation}

CST results and measurements performed with the D-dot sensor, both confirmed that the amplitudes of the electric field waveforms are consistent with the expected dipole beam pattern. In such conditions, the total radiated energy per pulse can be estimated from the time domain electric field waveform $E(t)$ measured at $\theta=\pi / 2$ and at a radial distance $R=1.2 \mathrm{~m}$ (see Fig. 3) as:

$$
W=\int_{0}^{\pi} \int_{0}^{2 \pi} \int_{0}^{\infty} \frac{E(t)^{2} R^{2}}{\eta_{0}} \sin ^{3}(\theta) d \theta d \varphi d t
$$

where $\eta_{0}$ is the free space impedance. Performing the calculations, the result is $W=25.6 \mu \mathrm{J}$. As the initial energy stored in the inter-electrode capacitance at a charging voltage of $5 \mathrm{kV}$ is $83.1 \mu \mathrm{J}$, the efficiency of generating electromagnetic radiation with a spectrum up to $3.5 \mathrm{GHz}$ is about $31 \%$.

\section{Discussion of numerical modeling results}

Both the PSpice and the CST analysis suggest the dielectric breakdown switch closes in a few ps. This characteristic favorably compares with the fastest closing switches in use in high-voltage pulsed power technology [10], [11].

It is interesting to compare this result with an estimate using the well-known Charlie Martin formula, valid for solid dielectric breakdown nanosecond switching [12]. According to this seminal work, the total effective rise time of the pulse $\tau_{\text {tot }}$ (in ns), is controlled by a combination of an inductivephase term $\tau_{\text {ind }}$ and a resistive-phase term $\tau_{\text {res }}$ : 

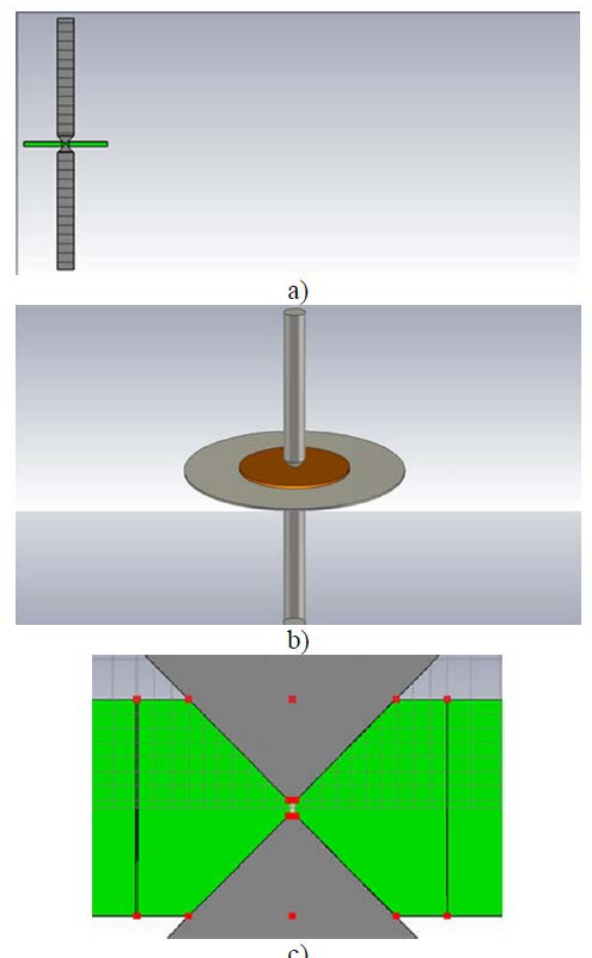

Fig. 11. CST model of the UMS: a) general overview, b) details of the source and c) zoomed view, highlighting the fine mesh in the region near the HV electrode tips.

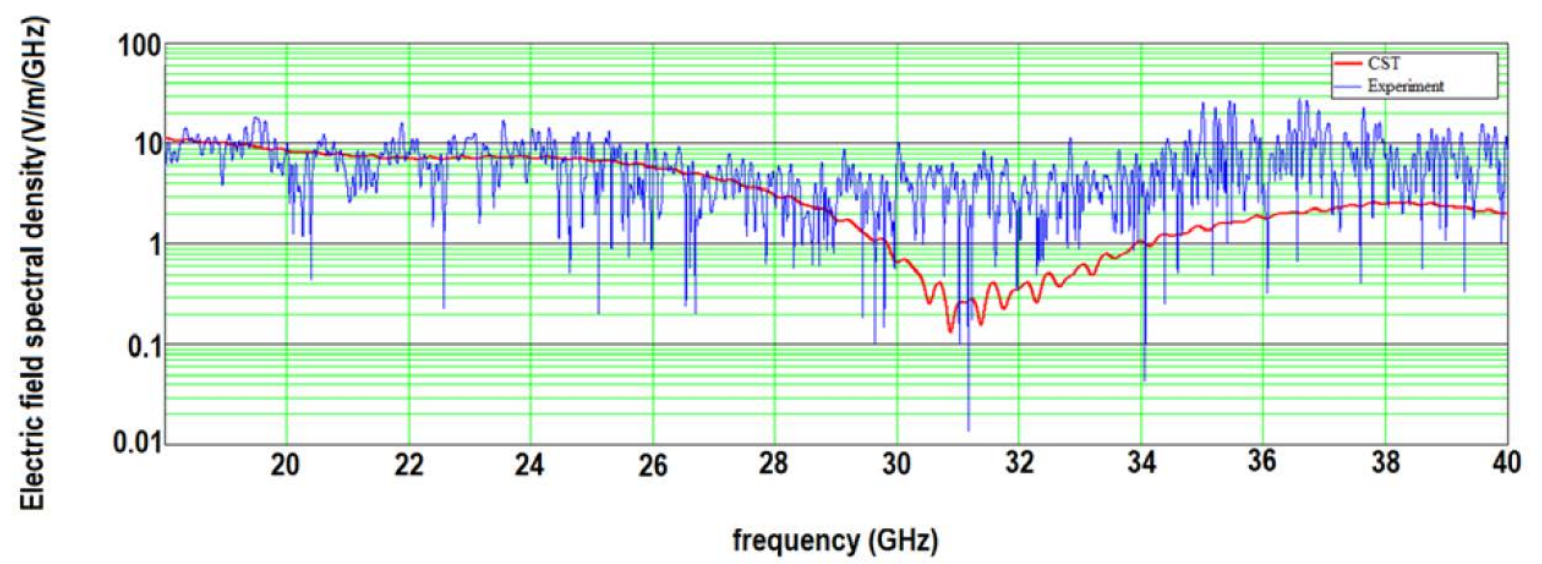

Fig. 12. Spectral density calculated with CST and compared with experimental data. The microwave radiation was detected with a $40 \mathrm{GHz}$ horn antenna (Flann DP241) positioned $1.4 \mathrm{~m}$ from the HV electrode. The real time digital oscilloscope used to record the signal has an analogue bandwidth of $65 \mathrm{GHz}$ with $160 \mathrm{GS} / \mathrm{s}$.

$$
\tau_{\text {tot }}=\tau_{\text {ind }}+\tau_{\text {res }}=\frac{L(l)}{Z}+\frac{5}{Z^{\frac{1}{3}} E^{\frac{4}{3}}}
$$

where $L(l) \approx 14 l$ is the self-inductance of a plasma channel (in $\mathrm{nH}$ ) having a length $l$ (in $\mathrm{cm}$ ). $E$ is the electric field breakdown (in $\mathrm{MV} / \mathrm{cm}$ ) and, adapting for our case, $Z$ is the impedance of the antenna (in $\Omega$ ). The type of closing switch used in this work has been extensively used in the past by the AWE pulsed power group and it was hypothesized [12] that in the extreme case the polyethylene intrinsic electric field breakdown may reach values as high as $E=8 \mathrm{MV} / \mathrm{cm}$. For our case the length of the plasma breakdown channel can be therefore estimated as $l \approx \frac{V}{E}=6.25 \cdot 10^{-4} \mathrm{~cm}$ (about $6 \mu \mathrm{m}$ ), where $V=5 \cdot 10^{-3} \mathrm{MV}$ is the initial charging voltage. With the antenna impedance estimated as $Z \approx 120 \Omega$ and the selfinductance calculated as $\mathrm{L}=8.75 \mathrm{pH}$, the switching time can be estimated as:

$$
\tau_{\text {tot }}=\frac{8.75 \cdot 10^{-3}}{120}+\frac{5}{120^{\frac{1}{3} 8^{\frac{4}{3}}}}=0.063 \mathrm{~ns} \quad \text { or } \quad 63 \mathrm{ps} .
$$

Apparently, (5) is not valid for the extremely fast UMS switching process.

\section{CONCLUSIONS AND THE WAY AHEAD}

The extreme simplicity and compactness of the UMS practical arrangement, when compared with the unique results that it generates, is certainly striking. In the future, the 
UMS phenomenon may find application in the characterization of the electric breakdown speed in dielectrics.

Planned future research, using $100 \mathrm{GHz}$ instrumentation, will investigate if the UMS produces radiation in a higher frequency bandwidth. Other suggested ways ahead are to use an explosively-driven electrode in an effort to investigate the UMS phenomenon using ceramic materials while at the same time possibly charging the system to a much higher voltage.

\section{ACKNOWLEDGMENT}

The authors wish to express their gratitude to Teledyne LeCroy for their interest in this work and their cooperation in making available to the authors the Labmaster $10 \mathrm{Zi}$, a state-of-the-art $65 \mathrm{GHz}$ real time digital oscilloscope at a time when the unit was in great demand.

Special thanks goes to Dr. Jiahui Yin, Xi'an Jiaotong University, Xi'an, P.R. China, for his contributions to the PSpice model.

\section{REFERENCES}

[1] H. Kolsky, "Electromagnetic Waves Emitted on Detonation of Explosives”, Nature, vol.173, p. 77, Jan. 1954.

[2] A. Misra, "Electromagnetic effects at metallic fracture", Nature, vol.254, pp. 133-134, March 1975.

[3] C. G. Camara, J.V. Escobar, J.R. Hird and S.J. Putterman, “Correlation between nanosecond X-ray flashes and stick-slip friction in peeling tape”, Nature, vol.455, pp. 1089-1093, Oct. 2008

[4] C. J. Buchenauer and J. R. Marek, "Hybrid antenna-sources for radiating high-power impulsive fields," Proc. SPIE, vol. 2557, pp. 209-213, 1995.

[5] X. Song, G. Liu, Y. Fan, X. Liu, and F. Liu, "Antenna-source integrated ultra-wideband electromagnetic pulse radiating device," Ultra-Wideband Short-Pulse Electromagn., vol. 4, pp. 145-148, 1999.

[6] H. G. Roskos and M. D. Thomson, "Ultra-broadband THz pulses From millimeter waves to the infrared", Lasers and Electro-Optics Europe (CLEO EUROPE/IQEC), International Quantum Electronics Conference, p.1/1, 2013.

[7] M. Tani, M. Herrmann and K. Sakai, "Generation and detection of terahertz pulsed radiation with photoconductive antennas and its application to imaging”, Meas. Sci. Technol. vol. 13, pp. 1739-1745, 2002

[8] B. M. Novac, S. W. Braidwood, I. R. Smith and M. Hubbard, "Detection of broadband microwave radiation from a solid-dielectric closing switch”, Meas. Sci. Technol.,vol.15, pp. L11-L14, Sep. 2004.

[9] C. G. Garton and N. Parkman, "Experimental and theoretical investigation of conduction in polyethylene from $4 \mathrm{MV} / \mathrm{m}$ up to 'intrinsic' breakdown”, Proc. IEE, vol.123, pp. 271-276, Jan. 1976.

[10] Picosecond Optoelectronic Devices, Edited by H. Lee Chi, Elsevier Inc., 1984.

[11] FID Gmbh, accessed February 2018. [Online]. Available: http://www.fidtechnology.com/products/fpg-s-picosecond.html

[12] J. C. Martin, "Nanosecond Pulse Techniques", Proc. IEEE, vol. 80, No. 6, pp. 934-945, June 1992.

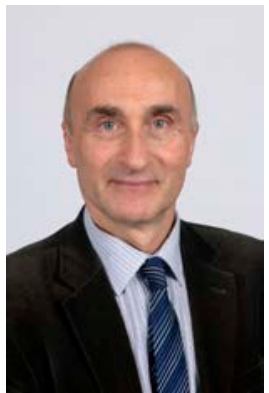

Bucur M. Novac (M'06 - SM'08) received the M.Sc. and Ph.D. degrees in 1977 and 1989, respectively, both from the University of Bucharest. He joined the Loughborough University, UK in 1998 and is currently Professor of Pulsed Power. His research interests include compact and repetitive high-power systems, explosively and electromagnetically driven magnetic flux compression generators and their applications, electromagnetic launchers, ultrafast magneto and electro-optic sensors and 2-D modeling of pulsed-power systems. He has coauthored two books on explosive pulsed power and has published more than 200 refereed papers and conference contributions.

Prof. Novac is a voting member of the Pulsed Power Science \& Technology Committee in the IEEE Nuclear and Plasma Science Society. He is also a member of the International Steering Committees for both the MEGAGAUSS Conferences and for the Euro-Asian Pulsed Power Conferences. He is also member of the organizing committee for the IEEE International Power Modulator and High Voltage Conference and cochairman of the UK Pulsed Power Symposium. Prof. Novac is a Chartered Engineer and a Fellow of both the Royal Academy of Engineering and the Institution of Engineering and Technology, UK.

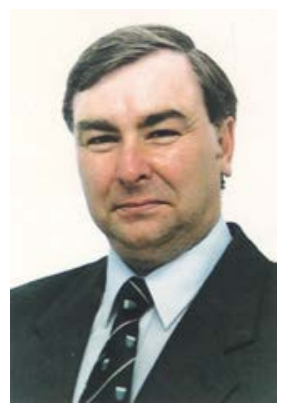

Peter Senior (M’16) received an Honours Degree in Physics with Electronics from Leicester University. He is a member of the Institute of Physics and a Chartered Physicist. He began his career in the Electronic and Electrical Engineering Department at Loughborough University researching Ultrasonic Nondestructive Evaluation, extending into Nonlinear (Finite Amplitude) Acoustics. He was one of the founder members of the Pulsed Power Research Group. He has worked on Propellant and Explosive Pulsed MHD Generators, and also produced systems to support a Flux Compressor Programme. Other work has included High Efficiency Launchers and the Electromagnetic Protection of Armoured Vehicles. He has produced a number of Transportable High Energy, High Power Systems, varying in size between fitting into a van, and up to two ISO Containers, supplied by diesel generators. This has required expertise in the Health and Safety Aspects of integrating Pulsed Power systems with the equipment of sponsoring and collaborating bodies.

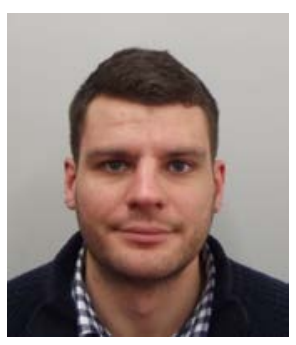

Michael Farage received an MEng in Electronic and Electrical Engineering from Loughborough University, Leicestershire, U.K in 2016 during which he completed an industrial placement year as an electronics engineer with GE Groby, Leicestershire, U.K. He is currently pursuing a $\mathrm{PhD}$ in Electronics and Electrical Engineering at the University of Glasgow. His research interests include the design, fabrication and characterisation of components for submillimetre wave imaging and radar applications.

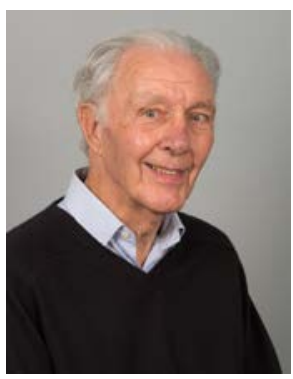

Ivor R. Smith (M'05-SM'11) received both B.Sc. and Ph.D. degrees from the University or Bristol, UK after completing an indentured student apprenticeship at the Witton Works of the General Electric company. He then became a Lecturer at the University of Birmingham, UK, subsequently being promoted to Senior Lecturer and Reader and being awarded the degree of D.Sc. by the University of Bristol for his continued research contribution. He then moved to Loughborough University, UK to become Professor of Electrical Power Engineering, and served as Head of Department, Dean of Engineering and Pro-Vice Chancellor. For more than 25 years he has been active in research in many aspects of the production, conditioning and utilization of large pulses of electrical energy and his work has brought in very substantial funding from a variety of sources. 
Professor Smith is a Chartered Engineer and a Fellow of both the Institution of Engineering and Technology and the Royal Academy of Engineering.

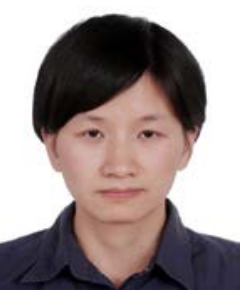

Renzhen Xiao received the Ph.D. degree in nuclear science and technology from Tsinghua University, Beijing, China, in 2007.

Since 2002, she has been with the Northwest Institute of Nuclear Technology, Xi'an, China. Her research interests include relativistic electronics and high power microwave generation and transmission. 